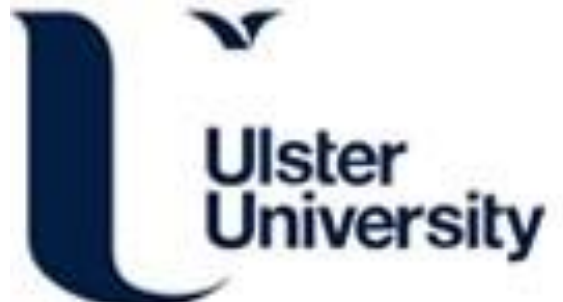

Towards Intelligent and Self-Evolving Network Infrastructures for Energy Management

Baumgarten, M., \& Mulvenna, M. (2010). Towards Intelligent and Self-Evolving Network Infrastructures for Energy Management. In Proceedings Fourth International Conference on Self-Adaptive and Self-Organising Systems - Workshop on Socio-Economics Inspiring Self-Managed Systems and Concepts (SEISMYC-2010) (pp. 72-75). IEEE Computer Society. https://doi.org/10.1109/SASOW.2010.17

Link to publication record in Ulster University Research Portal

\section{Published in:}

Proceedings Fourth International Conference on Self-Adaptive and Self-Organising Systems - Workshop on Socio-Economics Inspiring Self-Managed Systems and Concepts (SEISMYC-2010)

Publication Status:

Published (in print/issue): 01/10/2010

DOI:

10.1109/SASOW.2010.17

\section{Document Version}

Publisher's PDF, also known as Version of record

\section{General rights}

Copyright for the publications made accessible via Ulster University's Research Portal is retained by the author(s) and / or other copyright owners and it is a condition of accessing these publications that users recognise and abide by the legal requirements associated with these rights.

\section{Take down policy}

The Research Portal is Ulster University's institutional repository that provides access to Ulster's research outputs. Every effort has been made to ensure that content in the Research Portal does not infringe any person's rights, or applicable UK laws. If you discover content in the Research Portal that you believe breaches copyright or violates any law, please contact pure-support@ulster.ac.uk. 


\title{
Towards Intelligent and Self-Evolving Network Infrastructures for Energy Management
}

\author{
Matthias Baumgarten, Maurice Mulvenna \\ TRAIL Living Lab, School of Computing and Mathematics \\ Faculty of Computing and Engineering, University of Ulster \\ Shore Road, Newtownabbey BT37 0QB, United Kingdom \\ \{m.baumgarten, md.mulvenna\}@ulster.ac.uk
}

\begin{abstract}
Energy demands are constantly increasing worldwide and forecasts estimate them to rise by approximately $44 \%$ worldwide by $2030^{1}$. At the same time traditional energy sources such as oil, coal gas, etc. diminish continuously, and consequently, there is a demand for renewable clean energy that is closely tied with a general need for more efficient energy production, usage and distribution strategies. In particular, the use of micro-power plants that are, for example, incorporated into smart homes or public buildings offer great potential by providing more dynamic yet dependable energy supply mechanisms. Nevertheless, in order to maximize such resources it is necessary to correlate energy demand with potential supply mechanisms efficiently in order to reduce energy overheads and to maximize the use of available resources in general. Moreover, it is necessary to take into account individual social as well as economic aspects in order to model the underlying environment and the dynamics thereof accurately. For that, new mechanisms are required that lead to intelligent and self-managing networks of knowledge that have the capability to self-organize energy management according to various operational, spatial and socio-economic aspects such as demand, costs, consumer preferences, business goals and others. From an Information and Communications Technology (ICT) perspective, this paper discusses some of the requirements of next-generation energy grids and identifies some of the tasks and challenges in this area that need to be addressed in order to realize infrastructures that are fully intelligent and are also aware of their social and economic parameters so that energy usage can be dynamically and autonomously controlled in relation to global demand and supply.
\end{abstract}

Keywords: Autonomic; Self-Awareness; Self-Organsiation; Distributed and Intelligent Systems; Smart Grid

\section{INTRODUCTION}

The energy grid of the future is one of the most important and at the same time most difficult visions for today. It comprises micro- as well as large-scale energy resources, together with millions of end users and billions of devices that need to be coordinated and controlled based on individual demand and supply levels and in relation to specific consumer preferences and business needs. Moreover, distinct social as well as economic aspects such as country specific laws and regulations, environmental considerations, user or business-orientated contexts and

\footnotetext{
${ }^{1}$ http://www.reuters.com/article/idUSN2719528620090527
}

others need to be incorporated into the overall management of such networks to fully reflect the dynamic use in a real world context. In short, the energy network of the future needs to provide more than energy on its own - it includes the control and organization of the on-demand production, efficient distribution and optimized use of clean and renewable energy on a global scale.

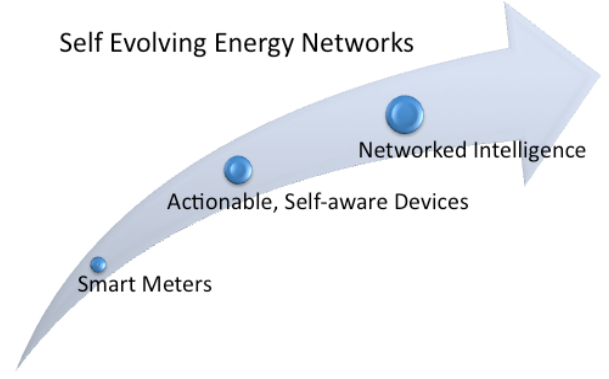

Figure 1. Towards Self-Evolving Smart Grids

As depicted in Figure 1, such a bold vision cannot be achieved in a single step but requires a number of stages that will eventual lead to highly dynamic, intelligent and, ultimately, self-evolving networked infrastructures that are self-aware of themselves and that have the ability to monitor and control virtually every aspect of themselves but in particular the devices that consume and produce energy as well as the context in which they are used. The introduction of smart meters can be seen as the first step towards this vision, which will allow the detailed monitoring of consumed energy also providing feedback mechanisms to better match overall consumption with generation. In addition, they will enable more dynamic, time-of-use pricing / billing models that will encourage overall energy reduction. This aspect in particular introduces new dimensions in which business objectives can actively influence consumer characteristics and vice versa. The second step towards self-evolving energy infrastructures is formed by the need that virtually all aspects thereof need to be self-aware and actionable. That is, that each device that consumes / produces energy is aware of its own status and context of use and is also controllable in a way that overall energy demand can be adjusted based on the available supply and, at the same time, energy supplies can be adjusted based on current or estimated demands. This will require rapid and efficient mechanisms that firstly, recognize the changes in demand and, subsequently, identifies and activates or deactivates suitable supply channels in dependence of their 
context, e.g., energy type, costs, spatial distance, efficiency, etc.; or alternatively, decrease demand by deactivating devices or by delaying the activation in a context-aware manner.

Considering the scale and the complexity of energy networks as a whole, the above can only be achieved if future systems operate in a fully decentralized fashion on environments that are properly understood on a structural level as well as in the way they are utilized over time. For this, a new kind of 'perpetual' contextualization mechanism is required that is capable of perceiving all aspects of the networked environment and their use in a machine understandable and pervasive fashion, which is maintained in real time and which emerges from the networked intelligence that needs to be interwoven with the environment itself. In turn, this will provide the knowledge base on which decision-making can be performed thus allowing the autonomous self-management at various levels of granularity.

The remainder of this paper is organized as follows. Section II discusses relevant requirements and outlines some possible research directions in relation to the identified challenges, while Section III concludes the paper.

\section{REQUIREMENTS AND RESEARCH DIRECTIONS}

The smart grid of the future reflects a highly complex, heterogeneous network of virtually unlimited dimensions also embracing an immeasurable number of components that exist at various granular levels. However, in contrast to the existing electrical grid it is envisioned that the future smart grid is fully self-managing aiming at reducing overall energy consumption, increase energy efficiency and to optimize energy distribution in order to reduce costs, emissions and other negative aspects. This requires that the intelligence that facilitates individual management tasks is closely interwoven with the distributed infrastructure and practically embedded into the components thereof. For this vision to be become reality, all aspects of the grid, that is, all devices that consume or produce energy as well as the components that deal with the storage or distribution of energy, need to have the following characteristics:

Self-awareness: That is that individual components are aware about their own purpose, properties, context of use as well as about the environment in which they exist [6]. As such, devices need to develop their own individual "identity" which needs to be capable to reflect all contextual attributes as well as their general use characteristics. This would allow components to express their demands as well as the services or supplies they offer to either themselves or to other stakeholders. For instance, devices need to be energyaware in relation to their use such that they are aware of their energy demands over time, e.g., a television unit is usually on stand-by for most daylight and late-night hours but is often switched on for evening hours or for popular events. Modeling and monitoring the energy demands of individual devices over time would allow to predict future demands at different granular levels which in turn could be used to predict and control energy supplies. This, however, requires each device to be aware of how much energy it is consuming, when it is on and also when it is likely to be on or how it is used, e.g., some devices always use a relative constant amount of energy whereas for others this may vary depending on how they are used.

Actionable: Within the context of this work, actionable means that a component can adjust its own operational parameters either by itself or through external stimuli or advice; for example, individual components may autonomously activate themselves in dependence of relevant contextual parameters, or devices may deactivate themselves for a short period if available energy levels drop below a certain threshold. Alternatively, other constraints that e.g., involve the coordination of ensembles of devices may be modeled into a conceptually, higher orientated management or supervision layer that efficiently manages specific clusters of devices that are self-organized based on individual social or economic parameters, e.g., a fridge and a freezer unit may be activated in an alternating fashion thus reducing energy peaks that occur if they would be activated simultaneously. Alternatively, and in relation to components that generate or distribute energy, they could be dynamically (de-)activated or configured, respectively, to serve energy in an on-demand fashion thus reducing overheads by better correlating the amount of energy produced to the amount of energy that is actually needed at any given, or even more compelling predicted moment in time. While this problem seems trivial on a per household basis it represents a considerable challenge on the global network as energy demand can increase drastically within very short periods of time. E.g., [1] reported that energy demand surged during a football match by 2800 megawatts in the UK alone, which reflected an increase of approximately $10 \%$ of previous demand. This points out that energy usage is, at least in the private sector, largely correlated to social behavior or activities such as working and living patterns. In fact and as highlighted in [1] and [2], most common energy surges are actually related to social activity such as particular popular TV events in a way that energy demands rise whenever they are broadcast but often surge dramatically during breaks or right after the end of a program. While this is not surprising as it is normal behavior for people to engage in additional activity during, e.g., an advertising break, it does pose severe challenges on the current network and the quality of service thereof. On the other hand, it provides the opportunity to model such activity into the use characteristics of a self-aware device or the home management system of a smart home itself, which in turn can propagate such information to be analyzed within the overall networked infrastructure in order to activate or deactivate individual resources as needed. Thus, selfawareness is unarguably a prerequisite for actionable infrastructures.

In relation to Figure 1, each of the discussed aspects reflect the requirements for the second step towards self-evolving infrastructures as they would allow for (a) more fine-grained monitoring of energy demands, which is not only based on the actual energy consumption on a per-device, -house, -city, -county, etc. level but would also include detailed use-characteristics and (b) provides 
actionable interfaces to control and as such manage individual components in order to self-manage overall demand and supply. Nevertheless, through the above, devices can only be managed and controlled on a per component basis. That is, that each component only exists in isolation and, even if capable of communicating with other components, efficient self-organization could not yet be achieved on a global scale where the network itself would need to be aware of all the components it embraces and in particular their energy demands and producing capabilities. Such intelligence, that would be required for the overall network to become self-managing, needs to be embedded into the infrastructure itself in order to have real time access to the underlying knowledge sources on which self-organization and decision making can be performed on. This reflects the final step towards self-evolving infrastructures and would provide the means to correlate and to control energy demand / supply at different conceptual or spatial levels thus providing the framework in which selforganized behavior can emerge from. In order to facilitate such networked intelligence that, by definition, needs to be fully distributed and decentralized, a number of challenges need to be addressed of which some are discussed next.

Complexity: The complexity of the data required to model self-awareness forms a key challenge and is based on the degree of self-awareness to be modeled, the diversity of the components involved and the relation they have to each other. Furthermore, the mapping and correlation of individual data / devices to different contexts implies a virtually unlimited complexity that is obviously difficult to model and to maintain. Modeling a system at various granular levels in dependence of their individual requirements provides a means to deal with the complexity by varying the level of detail for which a device or system is modeled or analyzed. For instance, a smart home could be looked upon from the outside as a black box hiding individual devices and their profiles only propagating synopses or aggregates that are required. Alternatively it could be looked upon as a more fine-grained device or even at the device-attribute level where each device or their attributes is exposed to the services outside the scope of the smart home.

Conceptual aspects that need to be modeled into the self-aware profile of a smart grid component (SGC) for it to become self-aware about itself and for it to become manageable from other services include its context of use, its business context, its energy demand / supply profile and its neighborhood which refers to the devices and contexts with which it has a relation. In order to deal with the volume, dimensions and complexity of the data involved to maintain such a profile, flexible feature extraction and other data reduction mechanisms are required that reduce subsequent communication and processing load. Moreover, such mechanisms may also be used to translate raw data into more meaningful, semantic attributes, which can be better interpreted at subsequent stages. This would allow for the semantic modeling of various aspects such as spatial, temporal, user as well as business specifics, which are required to construct and maintain complex self-aware profiles that include operational, social as well as economic dimensions and as such provide the knowledge base upon which self-evolution can be based.

Self-Evolving Data and Knowledge Management: Large-scale intelligent infrastructures are characterized to be highly decentralized and heterogeneous, which contradicts with many of the current monitoring and analysis techniques that often reflect centralized approaches to which data is communicated for further analysis. As a consequence, any monitoring or processing needs to be performed in a highly decentralized fashion, preferably at the point where data is collected or where actionable decisions are applied at to the underlying environment. This becomes particularly evident when considering the amount of data to be monitored and analyzed and the speed at which this needs to be performed.

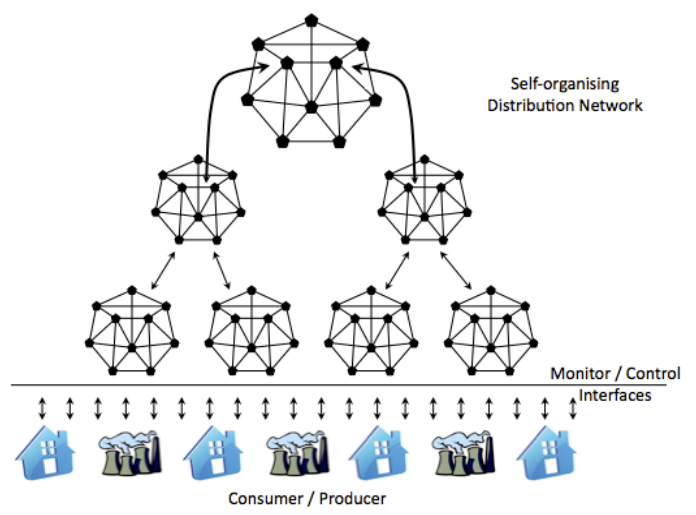

Figure 2. Self-Organizing SGO's

As depicted in Figure 2, embedding the required mechanisms for monitoring and data pre-processing tasks into the devices and the networked infrastructure offers new means to tackle the complexity of future systems by providing hierarchical or network like constructs to which relevant knowledge can be aggregated or propagated too. In such a reference-based framework, individual components are reflected by their SGC profile of which individual attributes can be used for self-organization. Thus, the realization and dynamic orchestration of such monitoring and knowledge extraction concepts should enable the (a) facilitation of different monitoring tasks at various levels of granularity; (b) handling of different complexity levels to accommodate for multi-grained technical as well as social or business related contexts; and (c) facilitation of intelligent knowledge diffusion mechanisms to avoid the broadcasting of information which inevitably would result in an communication as well as information overload. The resulting framework should enable on the fly knowledgeextraction, -diffusion and -reasoning over complex data, which includes the devices themselves, the interactions among them and the services operating on them. Similar concepts have already been discussed by Clark et al. [7] in relation to the Internet or Bicocchi et al. [6] through the concept of knowledge networks which reflect a knowledge management layer that extracts knowledge from a lower orientated data layer providing it to a higher orientated services and application layer in a well structured and usable way. 
Knowledge Reasoning: The autonomous establishment and adaptation of relations between stakeholders, knowledge and services as well as the verification of individual contexts in relation to given parameters will depend heavily on flexible and adaptive reasoning mechanisms. This is particularly relevant when considering that in order to reason about the control or optimization of energy demands, any such mechanism needs to be aware about the type, purpose and context of the device(s) concerned and the relations among them. This is to avoid unwanted or dangerous situations that could result if devices are (de-) activated without taking the current context of use into account. For instance, deciding to switch off a light while someone is walking down some stairs could be considered as dangerous.

A number of reasoning principles have been proposed by Turner et al. in [5] and have been categorized to be inductive, deductive or abductive where deductive reasoning, being a top-down approach allows to validate or invalidate a theory or condition, which can be used for the generation of vertical overlays where generalized knowledge can be modeled at specific granular levels. Inductive reasoning on the other hand is a bottom-up approach and seeks a broader generalization that is based on specific observations, which is of particular interest for the discovery of relations between stakeholders. Finally, abductive reasoning attempts to infer logical explanations that are based on observations. This is of interest when generating assumptions or for the extraction of descriptive explanations for the benefit of better humancomputer or automated computer-computer interactions.

Responsiveness: The responsiveness of a system indicates how quickly it can adapt to changing conditions, which could, if it is too slow, render a system to be useless. This aspect is highly relevant within this context as a continuous quality of service is irrefutably the highest priority for any energy network. Nevertheless, considering the speed, volume and complexity at which data must be correlated, analyzed and reacted upon, traditional knowledge extraction and inference mechanism that do not operate in real-time or that require large knowledge bases may not prove to be sufficient. However, latest advances in stream based data mining and knowledge extraction techniques such as discussed in [4] could analyze data on the fly.

\section{Towards Predictive Capabilities:}

chareteristic relevant for the self-evolution: infrastrustic relevant for the self-evolution of networked infrastructures is that of prediction, which could lead towards proactive components and interfaces that go some way towards the provision of a 'calm' environment, as envisaged in [3]. Such mechanisms provide the ability to predict the possible future context of devices and device ensembles thus allowing prediction of time and the amount of energy needed. This means that, based on the observation and analysis of past behavior and the use of predictive reasoning, a system could predict its own future states for various aspects of e.g., its own operational environment to either guide itself to a more optimum state or, if necessary, to prevent unwanted or dangerous situations before they actually occur. This is of interest to smart energy grids as it would enable the (a) prediction of the energy demand required over time allowing pre-activation of necessary resources thus improving responsiveness; and (b) the provision of estimates on how much energy is available at future points in time through each unit or distribution point. The latter in particular is of interest for energy resources that are for various reasons not fully controllable such as wind, solar, wave, etc.

\section{CONCLUSIONS}

The vision of the smart grid is undoubtedly one of the great inter-disciplinary challenges of today that involves a multitude of challenges from different domains. Modeling the energy demands at very fine granular levels and also correlating them with the available supply is an important step towards generic energy optimization mechanisms in which smart infrastructures and the smart devices therein may be autonomously controlled in dependence of available energy supplies or the associated costs thereof. Moreover, the incorporation of socio-economic as well as environmental parameters into the organization and management thereof does provide additional potential that goes well beyond the possibilities of the current network. Similar, predicting energy demands at a very fine grained level could prove important as it would enable the efficient planning, usage and optimization of energy resources and their distribution. This emphasizes the need for conceptually new methodologies that allow to efficiently monitor, analyze and, ultimately, control the relations between energy demand $\Leftrightarrow$ energy distribution $\Leftrightarrow$ energy supply at all levels of granularity. This could help to bridge the gap between unsupervised and inefficient use of energy and complex coordinated energy-aware activities, which can be monitored, aided, guided and controlled at various levels and for different purposes.

\section{REFERENCES}

[1] Energy Demand; http://www.nationalgrid.com/NR/rdonlyres/ 1C4B1304-ED58-4631-8A84-3859FB8B4B38/17136/demand.pdf

[2] Derek W. Bunn, Jeremy P. Seigal; "Television peaks in electricity demand", Energy Economics, Volume 5, Issue 1, ISSN 0140-9883, pp $31-36,1983$

[3] M. Weiser; "The Computer for the $21^{\text {st }}$ Century", Scientific American, 265(3), 1991

[4] C. C. Aggarwal (Ed.); "Data Streams: Models and Algorithms (Advances in Database Systems)", Springer, ISBN 978-0387287591, 2006

[5] R. M. Turner; "Adaptive Reasoning for Real-World Problems: A Schema-Based Approach", Lawrence Erlbaum Associates, Inc., ISBN 9780805812985, 1994

[6] N. Bicocchi, M. Baumgarten, N. Brgulja, R. Kusber, M. Mamei, M.D. Mulvenna, F. Zambonelli; "Self-organized Data Ecologies for Pervasive Situation-Aware Services: The Knowledge Networks Approach", IEEE Transactions on Systems, Man, and Cybernetics, Part A: Systems and Humans, Vol. 40, pp 789 - 802, 2010

[7] M. Baumgarten, D. Guldenring, M. Poland, C. Nugent, J. Hallberg; "Embedding Self-Awareness into Objects of Daily Life - The Smart Kettle", Proceedings of the 6th International Conference on Intelligent Environments, DOI 10.1109/IE.2010.50 pp 34 - 39, 2010

[8] D.D. Clark, C. Partridge, J.C. Ramming, J.T. Wroclawski; "A knowledge plane for the internet"; Conf. on Applications, Technologies, Architectures, and Protocols for Computer Communications, pp. 3 - 10, 2003. 